Background: Aminaphtone (AMI) is a bioflavonoid compound, classically used for "capillary disorders". In vitro AMI interferes with adhesion molecules (sELAM-1 and SVCAM-1) and with vascular endothelial cadherin degradation thus defending vessels permeability. Moreover, it counteracts vasoconstriction, downregulating endothelin-1 production at a gene level (1-3). In vivo AMI ameliorates clinical symptoms of several clinical conditions, above all Raynaud's phenomenon (RP), either primary or secondary to systemic sclerosis (SSc), as demonstrated by a recent six-month study (4).

Objectives: To evaluate long-term tolerability of standard dosage of AMI in a real-life cohort of SSc patients with secondary RP.

Methods: Seventy-eight SSc patients (mean age $65 \pm 13$ years; mean disease duration $9 \pm 7$ years) treated with $\mathrm{AMI}$ due to active RP were enrolled (ACR/ EULAR 2013 criteria). They were taking various concomitant treatments, including aspirin, calcium-channel blockers, cyclic intravenous iloprost, immunomodulators, endothelin receptor antagonists. SSc patients performed periodic clinical assessments and blood tests on average every four months per clinical practice. Duration of AMI administration, side effects, and self-assessment of Raynaud Condition Score (RCS) in a scale from 0 (absence of pain) to 10 (intolerable pain) were retrospectively taken into account.

Results: Duration of AMI administration was between six and sixty-seven months (mean $31 \pm 20$ months). AMI was administered at $75 \mathrm{mg}$ bis in die dosage, as standard initial posology. At baseline, mean RCS was $7.3 \pm 0.8$. After 3 months of treatment sixty-four patients (82\%) yet referred a subjective improvement of $\operatorname{RCS}(3.5 \pm 0.8, p=0.03)$, whereas 14 patients $(18 \%)$ were clinically unsatisfied (RCS $6.1 \pm 0.4, p=1.12$ ). In this last group, posology was increased to $75 \mathrm{mg}$ tris in die, with a satisfactory amelioration in further nine patients $(93,6 \%)(\operatorname{RCS} 4.0 \pm 0.6 p=0.04)$, while five patients $(6,4 \%)$ definitively discontinued therapy for subjective ineffectiveness within six months. Patients referred a sustained improvement of RCS along the observational period (31 \pm 20 months) (last RCS $3.7 \pm 0.7, p=0.03$ vs baseline). During the follow-up, five patients $(6,4 \%)$ referred headache as side effect: three of them had to reduce $\mathrm{AMI}$ posology to $75 \mathrm{mg}$ per day, while maintaining clinical benefits. Periodic blood tests did not reveal any significant alteration attributable to AMI. No other side effects related to the drug appeared during the treatment period.

Conclusion: AMI shows an acceptable medium-long-term tolerability along with sustained efficacy in the management of SSc-related RP, without disabling side effects. However, the retrospective design, the absence of a placebo-control group and the concomitant standard therapy limit the results, and a randomized controlled trial for AMI use in the management of SSc-related RP is desirable.

References:

[1] Scorza R et al. 2008. Clin Ther 30(5):924-9.

[2] Felice F et al. 2018. Phlebology 33(9):592-599.

[3] Scorza R et al. 2008. Drugs R D 9(4):251-7.

[4] Ruaro B et al. 2019. Front Pharmacol 10:293

Disclosure of Interests: Emanuele Gotelli: None declared, Sabrina Paolino: None declared, Federica Goegan: None declared, Francesco Cattelan: None declared, Massimo Patanè: None declared, Carmen Pizzorni: None declared, Maurizio Cutolo Grant/research support from: Bristol-Myers Squibb, Actelion, Celgene, Consultant of: Bristol-Myers Squibb, Speakers bureau: Sigma-Alpha, Alberto Sulli Grant/research support from: Laboratori Baldacci DOI: 10.1136/annrheumdis-2020-eular.5050

\section{AB0579 ANTI-J01 ANTIBODIES IN A REAL-WORLD POPULATION.}

M. Greco $^{1,2}$, M. J. García de Yébenes ${ }^{3}$, F. J. Nóvoa Medina ${ }^{2}$, J. A. Hernandez Beriain $^{2}$, M. M. Riaño Ruiz ${ }^{2}$, M. J. Montesa ${ }^{4}$, I. Rua-Figueroa ${ }^{1}, E^{2}$ Loza $^{3}$, L. Carmona ${ }^{3}$. ${ }^{1}$ Hospital Universitario de Gran Canaria Dr. Negrín., Las Palmas de Gran Canaria, Spain; ${ }^{2}$ Hospital Universitario Insular de Gran Canaria, Las Palmas de Gran Canaria, Spain; ${ }^{3}$ Instituto de Salud Musculoesquelética Inmusc, Madrid, Spain; ${ }^{4}$ Hospital La Candelaria, Santa Cruz de Tenerife, Spain

Background: The presence of anti-aminoacyl transfer RNA synthetase (ARS) autoantibodies is essential for the antisynthetase syndromes (ASSD) diagnosis; as well, Anti-Jo1 ARS positivity is the highest weighted criterion in the 2017 EULAR/ACR criteria for idiopathic inflammatory myopathies (IIM).

Previous studies have shown differences in ARS specificity between different blot assays. Nevertheless, an adequate clinical correlation and its detection by another monospecific-assays or indirect immunofluorescence assay (IIFA), can safeguard it.

Objectives: To evaluate the prevalence of Anti-Jo1 ARS and their performance in the ASSD and IIM diagnosis criteria fulfillment in a real-world population.
Methods: We performed an observational retrospective study in one center in which IIFA and a blot assay (EUROLINE ANA Profile 3-IgG that include Anti-Jo1 but not other ARS) were performed in all cases with autoimmune disease suspicion (03/2007-07/2019).

We assessed: 1) Anti-Jo1 ARS prevalence, 2) The rate of Anti-Jo1 positivity in the performed blot assays, 3) The rate of patients with Anti-Jo1 ARS meeting ASSD or IIM criteria, and 4) The rate of true and false positive Anti-Jo1 ARS considering the IIFA and clinical correlation.

Results: A total of 419.361 inhabitants are under the center coverage area at the date, a total of 12.711 blot assays were performed during the observation period, and 61 cases presented Anti-Jo1 ARS positivity. The Anti-Jo1 ARS prevalence in the whole studied population was 0.00014 , representing $0.04 \%$ positivity of the performed blot assays.

Of those with Anti-Jo1 positivity:

- Only 4 patients (6.6\%) met Solomon's ASSD criteria and 26 (42.6\%) met Connors ASSD criteria (less strict), representing the $0.0009 \%$ and $0.006 \%$ of the whole population respectively.

- Five cases (8.2\%) presented a possible or probable IIM by Bohan and Peter criteria and 55 cases $(90.16 \%)$ presented a probable or definitive IIM by EULAR/ACR criteria, representing $0.001 \%$ and the $0.013 \%$ of the whole population respectively.

- The Anti-Jo1 positivity was not confirmed by a monospecific-assay. Nevertheless, 52 cases (85.25\%) presented positive IIFA (>1/80): 27 (51.92\%) nuclear, $12(23.08 \%)$ cytoplasmic and $12(25 \%)$ with both patterns. A total of 23 cases presented a fine speckled AC-19/AC-20 pattern; representing the $40.98 \%$ of all Anti-Jo1 ARS cases.

- Only 4 cases $(6.56 \%)$ did not meet any of the ASSD or IIM classification criteria. And only 4 cases (6.56\%) fulfilled Solomon ASSD criteria or presented a probable IIM by Bohan and Peter criteria, or a definite IIM by EULAR/ACR criteria. Thus, we can estimate that $6.56 \%$ of the cases were clinically false positives and other $6.56 \%$ were clinically true positive; leading a gap of $86.88 \%$ of cases with Anti-Jo1 ARS that only fulfill Connors ASSD criteria (less strict) or a not complete score to confirm the IIM diagnosis by Bohan and Peter or EULAR/ACR criteria.

Conclusion: The low prevalence of Anti-Jo1 ARS positivity observed and the low number of cases with confirmed ASSD or IIM in the Anti-Jo1 positive detected cases, suggest that:

- It is not efficient to test it by screening.

- It should be tested only under adequate clinical suspicion.

- Probably it is not convenient to include it in not myositis specific blot assays. Despite it is desirable to incorporate the evaluation of myositis specific antibodies in the IIM classification criteria; the differences observed between Bohan and Peter and 2017 EULAR/ACR criteria fulfillment in our series, suggest that the latter could be overweighting the Anti-Jo1 ARS positivity.

Disclosure of Interests: Martín Greco: None declared, María Jesús García de Yébenes: None declared, Francisco Javier Nóvoa Medina Speakers bureau: have been paid as a speaker for a few medical talks, José A Hernandez Beriain: None declared, Marta María Riaño Ruiz: None declared, María Jesús Montesa: None declared, Iñigo Rua-Figueroa: None declared, Estíbaliz Loza Grant/ research support from: Roche, Pfizer, Abbvie, MSD, Novartis, Gebro, Adacap Astellas, BMS, Lylly, Sanofi, Eisai, Leo, Sobi, Loreto Carmona Grant/research support from: Novartis Farmaceutica, SA, Pfizer, S.L.U., Merck Sharp \& Dohme España, S.A., Roche Farma, S.A, Sanofi Aventis, AbbVie Spain, S.L.U., and Laboratorios Gebro Pharma, SA (All trhough institution)

DOI: 10.1136/annrheumdis-2020-eular.6590

\section{$\mathrm{AB} 0580$}

\section{GENDER DIFFERENCES IN SYSTEMIC SCLEROSIS-} IMPACT ON DISEASE PHENOTYPE AND PROGNOSIS

L. Groseanu ${ }^{1,2}$, A. Balanescu ${ }^{1,2}$, V. Bojinca ${ }^{1,2}$, D. Opris-Belinski ${ }^{1,2}$, I. Saulescu ${ }^{1,2}$, D. Mazilu, ${ }^{1,2}$ S. Daia-lliescu ${ }^{1,2}$, A. Borangiu ${ }^{1,2}$, F. Berghea ${ }^{1,2}$, C. L. Constantinescu ${ }^{1,2}$, C. Cobilinschi ${ }^{1,2}$, M. M. Negru ${ }^{1,2}$, M. Abobului ${ }^{1,2}$, R. Ionescu ${ }^{1,2} .^{1}$ Carol Davila University of Medicine and Pharmacy, Bucharest, Romania; ${ }^{2}$ Sf Maria Clinical Hospital, Bucharest, Romania

Background: The low overall prevalence of systemic sclerosis (SSc) and the low proportion of male patients have resulted in a scarcity of studies assessing sex differences in SSc patients, and contradictory results.

Objectives: To evaluated sex influence on disease characteristics at baseline and then to estimate the effects of sex on disease progression and survival. Methods: We performed a retrospective observational study using data extract from the EULAR scleroderma trials and research (EUSTAR) cohort 096. 173 patients were analysed (26 males).

The severity of organ system involvement was defined as described previously (1) 
Results: Males were significantly older at symptom onset $(p=0.007)$ and at first center visit $(p=0.009)$. There were no differences regarding disease duration at first visit or the interval between the onset of Raynaud syndrome and other non-Raynaud manifestations $(p=0.06)$. Male patients were significantly more likely to have ever smoked $(p<0.001)$, males more often had severe or end-stage peripheral vascular involvement $(p=0.01)$. Modified Rodnan skin score (mRSS) was significantly higher in males $(p=0.004)$. We found no difference regarding musculoarticular involvement, except for digital contractures $(p=0.001)$ and tendon friction rubs $(p=0,044)$. Males more often had interstitial lung disease (ILD) $(p=0.013)$ which was also more frequently severe or end-stage $(p=0.003)$. Cardiac involvement was more common in males: pulmonary hypertension (PAH) $(p$ $=0.018)$, arrhytmias $(p=0.012)$, left ventricle ejection fraction $<45 \%(p=0.014)$. The frequency of scleroderma renal crisis $(S R C)$ was higher in males $(p=0.025)$. Gastrointestinal involvement did not differ between groups EScSG (European Scleroderma Study Group) disease activity scores were higher in males $(p=0.001)$. The isolated presence of antitopoisomerase-1 or anticentromere antibodies did not differ between groups. Mortality rate was similar between sexes, although male sex is a independent predictor for the death associated with ILD, SRC, arrythmias

In multivariate analysis, male sex was independently associated with a higher risk of diffuse cutaneous subtype (OR: 1.56, (1.35 to 1.84); $p<0.001$ ), a higher frequency of severe vascular disease (OR: 1.38 (1.11 to 1.67$) ; p<0.001)$, severe digital contractures (OR:1.92(1.68 to 2,42 ); $p<0.001$ ), interstitial lung disease OR: 1.22 (0.9 to 1.47$) ; p<0.001)$, severe heart involvement (OR: $1.56(1.22$ to 2,1$)$; $\mathrm{p}<0.001$ ) and SRC (OR: 3.31 (1.87 to 5620 ); $\mathrm{p}<0.003)$. In the longitudinal analysis, after a mean follow-up of $7.2( \pm 2.6)$ years, male sex was predictive of new onset of scleroderma renal crisis (HR: 3.66 (1.82 to 4.86); $p=0.006$ ) and heart failure (HR: 1.9 (1.36 to 3.18 ); $p=0.01$ ).

Conclusion: In essence, the disease prophyle in females is that of younger age of onset, longer disease duration at first center visit, less severe peripheral vascular involvement, the most frequent cause of death being $\mathrm{PAH}$. In contrast, males are older at onset, present earlier in their disease, have dcSSc, more severe peripheral vascular disease, higher mRSS, more frequent and severe ILD, more frequent heart involvement, higher risk of PAH and SRC, the most common cause of death being ILD. These results raise the point of including sex in the management and the decision-making process.

References:

[1] Peoples C, Medsger TA Jr, Lucas M et al Gender differences in systemic sclerosis: relationship to clinical features, serologic status and outcomes. J Scleroderma Relat Disord. 2016;1(2):177-240

Disclosure of Interests: Laura Groseanu Speakers bureau: novartis, eli-lilly, ucb, pfizer,sandoz, Andra Balanescu Consultant of: pfizer, Speakers bureau: Abbvie, Eli-Lilly, Novartis, Pfizer, Roche, Sandoz, UCB, Violeta Bojinca Speakers bureau: Eli-Lilly, Novartis, Pfizer, Daniela Opris-Belinski Speakers bureau: Abbvie, Eli-Lilly, Novartis, Pfizer, Roche, loana Saulescu Speakers bureau: Eli-Lilly, Pfizer, Diana Mazilu: None declared, Sanziana Daia-lliescu Speakers bureau: sandoz, Andreea Borangiu: None declared, Florian Berghea Paid instructor for: abbvie, Speakers bureau: gideon richter, egis, novartis, ucb, cosmin-laurentiu constantinescu: None declared, CLAUDIA COBILINSCHI Speakers bureau: novartis, Maria Magdalena Negru: None declared, mihai abobului Speakers bureau: gideon richter, Ruxandra lonescu Consultant of: Consulting fees from Abbvie, Eli-Lilly, Novartis, Pfizer, Roche, Sandoz, Speakers bureau: Consulting and speaker fees from Abbvie, Eli-Lilly, Novartis, Pfizer, Roche, Sandoz

DOI: 10.1136/annrheumdis-2020-eular.3350

\section{AB0581 HIGH PREVALENCE OF TUBERCULOSIS IN ADULTS AND CHILDREN WITH IIM AS COMPARED WITH SLE: RETROSPECTIVE DATA REVIEW FROM A LARGE COHORT AT A TERTIARY CARE CENTER IN INDIA.}

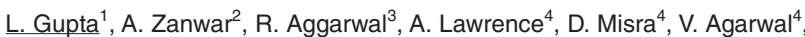
R. Misra ${ }^{5}$, A. Aggarwal ${ }^{4}$ on behalf of -. ${ }^{1}$ Sanjay Gandhi Postgraduate Institute of Medical Sciences, Clinical Immunology and Rheumatology, Lucknow, India; ${ }^{2}$ D Y Patil Hospital, Navi Mumbai, India; ${ }^{3}$ University of Pittsburgh Medical Center (UPMC), Division of Rheumatology and Clinical Immunology, 15260, United States of America; ${ }^{4}$ Sanjay Gandhi Postgraduate Institute of Medical Sciences, Lucknow, India; ${ }^{5}$ Kalinga Institute of Medical Sciences (KIMS), Bhubaneswar, India

Background: Infections are the most common cause of morbidity and mortality in idiopathic inflammatory myositis (IIM). India is endemic for Tuberculosis (TB) with a prevalence of 2.3 cases per thousand population.
Objectives: Thus, we studied the prevalence of TB in our cohort of IIM patients and compared with that in systemic lupus erythematosus (SLE).

Methods: Medical records from paper charts and electronic medical records were reviewed for adults and juvenile patients with SLE (ACR criteria 1997) and IIM (Bohan and Peter criteria 1975) first presented at a tertiary care hos pital in India from 1989 to 2016. Clinical variables including disease characteristics variables, the frequency, site, duration and complication of active TB as well as dose of corticosteroids and other immunosuppressive drugs were extracted retrospectively from the medical records. Descriptive statistics were used to describe the cohort and TB characteristics. Chi-square and t-test were used to evaluate association of TB with clinical diagnosis as well as medication data.

Results: There were 167 (132 adults and 35 juvenile) IIM and 280 (131 adults and 149 juvenile) SLE in our cohort. Active TB occurred in $24(14.4 \%)$ of all IIM cases $(18,13.6 \%$ adults; $6,17.1 \%$ juvenile) as compared to $18(6.4 \%)$ of all SLE cases $(8,6.1 \%$ adults; $10,6.7 \%$ juvenile, $p$ value $<0.01)$. Of all the TB in myositis most often it was seen in Dermatomyositis $(n=11,45.8 \%)$ followed by Polymyositis $(5,20.8 \%)$, and occasionally in Overlap myositis $(3,12.5)$ and juvenile dermatomyositis $(1,4.1 \%)$.

Considering an annual TB rate of 211 per 100,000 of the general population, the risk of developing active TB was 62-fold higher in patients with IIM and 27-fold higher in those with lupus. Patients with IIM had higher odds of developing TB as compared with Lupus [odds ratio 2.86 ( $\mathrm{Cl} 1.5-5.47), \mathrm{p}=0.007$ )

Amongst 24 IIM patients with TB, 10 had pulmonary TB and 14 had extra-pulmonary TB. The median glucocorticoid dose at the diagnosis of TB was $0.25(0-1.5) \mathrm{mg} / \mathrm{kg} /$ day. Half the cases of active TB occurred during inactive myositis. Seventeen patients with active TB were followed up over 27 months (8-184), with remission of TB in all cases but required prolonged courses of Anti-Tuberculous Therapy (ATT) in $25 \%$ cases with 10 ATT related adverse events in 8 patients and 5 patients with relapse of myositis due to lowering of immunosuppression.

Conclusion: Patients with IIM have higher prevalence of active TB as compared with SLE patients. The risk is highest in patients with Dermatomyositis possibly related to high doses of steroids. Extra-pulmonary forms of TB are more common, and patients commonly require prolonged course of ATT and may suffer relapses of myositis during ATT. Screening for latent TB may be useful in IIM patients before prescribing steroids and other immunosuppressive drugs.

References:

[1] TB Statistics India I National, treatment outcome \& state statistics [Internet] TB Facts I TB, tests, drugs, statistics. [cited 2019 Jun 13]. Available from: https://www.tbfacts.org/tb-statistics-india/

[2] Muhammed H, Gupta L, Zanwar A, Misra DP, Lawrence A, Agarwal V, Aggarwal A, Misra R;OPC0243: Infections are leading cause of in-hospital mortality in patients with inflammatory myositis; Indian J Rheumatol 2018;13, Suppl S2:93-241

[3] Gaitonde S, Pathan E, Sule A, Mittal G, Joshi VR. Efficacy of isoniazid prophylaxis in patients with systemic lupus erythematosus receiving long term steroid treatment. Ann Rheum Dis. 2002 Mar;61(3):251-3.

[18] He D, Bai F, Zhang S, Jiang T, Shen J, Zhu Q, et al. High incidence of tuberculosis infection in rheumatic diseases and impact for chemoprophylactic prevention of tuberculosis activation during biologics therapy. Clin Vaccine Immunol CVI. 2013 Jun;20(6):842-7.

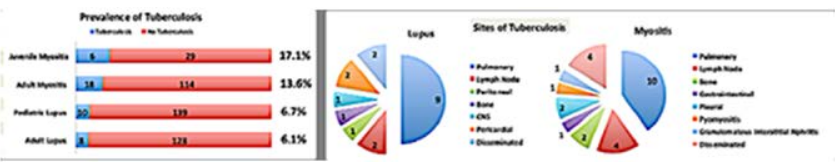

Figure 1. (A) Prevalence and (B) sites of tuberculosis

Table 1.

\begin{tabular}{|c|c|c|c|c|}
\hline \multicolumn{5}{|c|}{ Population demographics } \\
\hline Variable & Adult Lupus & Pediatric Lupus & Adult Myositis & Juvenile Myositis \\
\hline Patient number & 131 & 149 & $132^{\circ}$ & $35^{\prime \prime}$ \\
\hline Median follow up (years) & 3.0 & 6.0 & 5.5 & 8.0 \\
\hline Female : Male & $8: 1$ & 9:1 & 6.3:1 & 1.5:1 \\
\hline Median age (years) & 32.4 & 13.7 & 32 & 8 \\
\hline Lupus nephritis (\%) & 51.9 & 100 & NA & NA \\
\hline Chronic kidney disease (\%) & 4.6 & 7.3 & 0 & 0 \\
\hline Incidence rate/ 100 patient years follow up & 2.0 & 1.1 & 2.3 & 1.9 \\
\hline
\end{tabular}

